\title{
Markers for the Characterization of Liver Mesenchymal Stem Cell
}

\author{
Ahmed Lotfy ${ }^{1,3 *}$, Mennat Allah Abdelsamed ${ }^{1}$, Aya Abdelrahman ${ }^{1}$, Ahmed Nabil ${ }^{1,3}$, Ayman Abdel Fatah ${ }^{3}$, Walaa \\ Hozayen $^{1}$ and Gamal Shiha ${ }^{2,3}$ \\ ${ }^{1}$ Biotechnology and Life Science department, Faculty of Postgraduate Studies for Advanced Sciences, Beni-Suef University, Egypt
}

${ }^{2}$ I Department of Internal Medicine, University of Mansoura, Egypt

${ }^{3}$ Egyptian Liver Research Institute and Hospital (ELRIAH), Egypt

Submission: March 20, 2019; Published: April 17, 2019

*Corresponding author: Ahmed Lotfy, Biotechnology and Life Sciences Department, Faculty of Postgraduate Studies for Advanced Sciences (PSAS), Beni-Suef University, Egypt

\begin{abstract}
Mesenchymal stem cells (MSCs) show promise for use in regenerative medicine and tissue engineering. Bone marrow mesenchymal stem cells (BMSCs) are considered to be the standard, but MSCs can be isolated from many sources. Liver mesenchymal stem cells (LMSCs) are a promising alternative to BMSCs. One challenge in MSC research is immunophenotypic characterization of the cells, since there is no specific marker for MSCs to date; in addition, markers may differ among sources and among species. In this review, we will discuss the markers that have been tested on LMSCs from different species.
\end{abstract}

Keywords: Liver; Mesenchymal Stem Cells; Characterization; Bone Marrow; Human; Animals

\section{Introduction}

Mesenchymal stem cells (MSCs) have the characteristics of self-renewal, immune regulation and multipotency. Due to their multi-lineage differentiation potential [1], MSCs can be induced to differentiate into adipocytes, osteoblasts, chondrocytes, muscle cells, nerve cells, liver cells and pancreatic beta cells in vivo and in vitro. These characteristics make MSCs promising candidates for use in cell therapy and regenerative medicine [24]. MSCs were first described as stromal stem cells from the bone marrow that have a spindle shape in culture. However, although the bone marrow is considered the standard source for MSCs, MSCs isolated from other sources have different features in terms of surface markers, proliferation rates, and differentiation capability [5-7]. MSCs have been isolated from a number of other sources, such as adipose tissue, dental pulp, umbilical cord blood, and amniotic fluid [8-10]. One alternative source for MSCs is liver tissue. Scientists have isolated liver mesenchymal stem cells (LMSCs) from different species and attempted to characterize them in hopes that these cells could be a better alternative to bone marrow mesenchymal stem cells (BMSCs), especially for liver diseases [8]. One of the challenging aspects concerning MSCs in general is their characterization. In this mini-review, we will focus on studies that characterized LMSCs from different species, such as humans, mice, rats, rabbits, sheep, chickens and cattle.

\section{Human LMSCs and human BMSCs}

The International Society of Cell Therapy (ISCT) states that cultured human BMSCs are positive for expression of CD105, CD73, and CD90 and negative for expression of CD11b or CD14, CD19 or CD79a, CD34, CD45, and HLA-DR (11). CD105 is TGFbeta receptor III, which plays a role in TGF-beta signalling during differentiation into chondrocytes [12]. CD73 is known to be involved in MSC migration and to modulate adaptive immunity $[13,14]$.

CD90 plays roles in mediating cell-cell interactions and leukocyte adhesion to endothelial cells $[15,16]$. Human LMSCs are also positive for expression of CD105, CD73 and CD90 Table 1. However, Najimi et al. [17] reported that LMSCs have low expression of CD105, and most other studies reported that LMSCs are positive for CD105. Additionally, LMSCs are positive for expression of CD44, CD29, CD166, and CD146, which is consistent with BMSC expression patterns [18-20].

\section{LMSCs and BMSCs from other species}

In mice, LMSCs and BMSCs have nearly identical markers since both cell types are positive for CD44, CD29 CD105, CD49e, CD90 and Sca-1. However, mouse LMSCs are positive for CD73 [21], and mouse BMSCs have low expression of CD73 [22]. In rats, there are no studies on LMSCs; however, Payushina et al. [23] indicated that LMSCs are positive for expression of CD90. 
Jones et al. [24] reported that bovine BMSCs are positive for

expression of CD166 but positive for expression of CD44, CD29, expression of CD166. In addition, bovine LMSCs are negative for

CD73, CD90, and CD106 [25-43].

Table 1: Surface antigen expression on cultured LMSCs from different species.

\begin{tabular}{|c|c|c|c|c|c|c|}
\hline Surface Antigen & Human & Mouse & Rat & Rabbit & Chicken & Bovine \\
\hline CD44 & $\begin{array}{l}+(27) \\
++(17) \\
+(28) \\
+(29) \\
++(30) \\
++(31) \\
++(32) \\
+++(33) \\
++(34) \\
++(35) \\
++(36) \\
++(37)\end{array}$ & $++(21)$ & $++(38)$ & & $+(26)$ & $++(25)$ \\
\hline CD71 & & & & & $+(26)$ & \\
\hline CD29 & $\begin{array}{l}++(27) \\
++(17) \\
+(28) \\
+(29) \\
++(30) \\
++(31) \\
++(32) \\
++(33) \\
++(35) \\
++(36) \\
++(37) \\
+(39)\end{array}$ & $++(21)$ & $++(38)$ & & $+(26)$ & $++(25)$ \\
\hline CD73 & $\begin{array}{l}++(17) \\
+(28) \\
++(29) \\
++(30) \\
++(31) \\
++(32) \\
+++(33) \\
++(35) \\
++(36)\end{array}$ & $++(21)$ & $\begin{array}{l}+(23) \\
++(38)\end{array}$ & & $+(26)$ & $++(25)$ \\
\hline CD49e & $+(17)$ & $++(21)$ & & & & \\
\hline CD105 & $\begin{array}{l}-(17) \\
+(30) \\
++(31) \\
++(32)\end{array}$ & $\begin{array}{l}++(21) \\
++(40)\end{array}$ & $++(38)$ & & & \\
\hline
\end{tabular}




\begin{tabular}{|c|c|c|c|c|c|c|}
\hline & $\begin{array}{l}+++(33) \\
++(35) \\
++(36) \\
++(37) \\
++(41)\end{array}$ & & & & & \\
\hline CD14 & $\begin{array}{l}-(30) \\
+(32) \\
-(33) \\
-(37)\end{array}$ & $+/-(21)$ & & & & \\
\hline CD80 & & $+/-(21)$ & & $+(42)$ & & \\
\hline Ter119 & & $+/-(21)$ & & & & \\
\hline CD45 & $\begin{array}{c}+/-(27) \\
+/-(17) \\
-(30) \\
-(31) \\
+(32) \\
-(33) \\
-(34) \\
-(37) \\
-(39) \\
-(41)\end{array}$ & $\begin{array}{l}+/-(2) \\
++(40)\end{array}$ & & & & $-(25)$ \\
\hline CD86 & & $+/-(21)$ & & $-(42)$ & & \\
\hline CD133 & $\begin{array}{c}+/-(17) \\
-(30)\end{array}$ & $+(40)$ & & & & \\
\hline CD34 & $\begin{array}{l}+(17) \\
-(28) \\
-(30) \\
-(31) \\
-(32) \\
-(33) \\
-(34) \\
-(37) \\
-(41)\end{array}$ & $\begin{array}{l}+(40) \\
-(43)\end{array}$ & & & $-(26)$ & $-(25)$ \\
\hline . & $\begin{array}{l}++(27) \\
++(17) \\
+(28) \\
++(30) \\
++(31) \\
++(32) \\
++(33) \\
++(34) \\
++(35) \\
++(36) \\
++(41)\end{array}$ & $++(40)$ & $\begin{array}{l}++(23) \\
++(38)\end{array}$ & & & $++(25)$ \\
\hline
\end{tabular}


International Journal of Cell Science \& Molecular Biology

\begin{tabular}{|c|c|c|c|c|}
\hline Sca-1 & & $++(40)$ & & \\
\hline CG117 & $-(33)$ & $+(40)$ & & \\
\hline Thy-1 & & $-(43)$ & & \\
\hline c-kit & & $-(43)$ & & \\
\hline CD106 & $++(36)$ & & $+/-(23)$ & \\
\hline HLA Class I & $++(17)$ & & & \\
\hline CD13 & $\begin{array}{l}++(17) \\
+++(33)\end{array}$ & & & \\
\hline HLA-DR & $\begin{array}{c}+/-(17) \\
-(33) \\
-(41)\end{array}$ & & & \\
\hline $\mathrm{CD} 49 \mathrm{~b}$ & $+(17)$ & & & \\
\hline CD49f & $+(17)$ & & & \\
\hline MHC I & $+(31)$ & & $++(42)$ & \\
\hline MHC II & $\begin{array}{l}-(31) \\
-(35)\end{array}$ & & $+(42)$ & \\
\hline CD26 & $\begin{array}{l}++(31) \\
++(32)\end{array}$ & & & \\
\hline CD107 & $+(28)$ & & & \\
\hline CD117 & $\begin{array}{c}+/-(17) \\
-(28) \\
-(30) \\
-(31) \\
-(32) \\
-(33)\end{array}$ & & & \\
\hline CD166 & $\begin{array}{l}++(31) \\
++(32) \\
++(34) \\
++(35) \\
++(36) \\
++(37) \\
++(41)\end{array}$ & & $++(38)$ & $-(25)$ \\
\hline CD146 & $\begin{array}{l}+(30) \\
++(35) \\
++(36) \\
\end{array}$ & & & \\
\hline CD3 & $-(33)$ & & & \\
\hline CD4 & $-(33)$ & & & \\
\hline CD38 & $-(33)$ & & & \\
\hline CD39 & $-(36)$ & & & \\
\hline CD19 & $\begin{array}{l}-(33) \\
+(27)\end{array}$ & & & \\
\hline CD227 & $++(34)$ & & & \\
\hline CD54 & $++(36)$ & $++(38)$ & & \\
\hline
\end{tabular}

Symbols indicate marker expression levels: -: no expression; +/-: <5\% expression; +: 5-50\% expression, ++: 50-100\% expression. 


\section{International Journal of Cell Science \& Molecular Biology}

\section{Conclusion}

Many markers have been tested on LMSCs, especially on human and mouse samples, and BMSCs and LMSCs have been reported to share most markers. However, additional studies are required to establish a standard panel of positive/negative markers for LMSCs, and studies are also required for further characterization of LMSCs from other species, especially species that are used as in vivo models for many diseases.

\section{Data Availability}

The data used to support the findings of this study are available from the corresponding author upon request.

\section{Conflicts of Interest}

The authors have no conflicts of interest.

\section{References}

1. Dung Ngoc Khuu, Omar Nyabi, Cédric Maerckx, Etienne Sokal, Mustapha Najimi (2013). Adult Human Liver Mesenchymal Stem/Progenitor Cells Participate in Mouse Liver Regeneration After Hepatectomy. Cell Transplant 22(8): 1369-1380.

2. Guillot PV, Gotherstrom C, Chan J, Kurata H, Fisk NM (2007) Human first-trimester fetal MSC express pluripotency markers and grow faster and have longer telomeres than adult MSC. Stem cells 25(3): 646654

3. Tarnowski M, Koryciak Komarska H, Czekaj P, Sebesta R, Czekaj TM, et al. (2007). The comparison of multipotential for differentiation of progenitor mesenchymal-like stem cells obtained from livers of young and old rats. Folia histochemica et cytobiologica. 45(3): 245-254.

4. Todorov P, Hristova E, Konakchieva R, Michova A, Dimitrov J (2010) Comparative studies of different cryopreservation methods for mesenchymal stem cells derived from human fetal liver. Cell biology international34(5): 455-462.

5. Gotherstrom C, West A, Liden J, Uzunel M, Lahesmaa R, et al. (2005) Difference in gene expression between human fetal liver and adult bone marrow mesenchymal stem cells. Haematologica 90(8): 10171026.

6. El Kehdy H, Pourcher G, Zhang W, Hamidouche Z, Goulinet Mainot S (2016) Hepatocytic differentiation potential of human fetal liver mesenchymal stem cells: in vitro and in vivo evaluation. Stem cells international.

7. Baruteau J, Nyabi O, Najimi M, Fauvart M, Sokal E (2014) Adult human liver mesenchymal progenitor cells express phenylalanine hydroxylase. Journal of Pediatric Endocrinology and Metabolism 27(9-10): 863-868.

8. Herrero A, Prigent J, Lombard C, Rosseels V, Daujat Chavanieu M, et al. (2017) Adult-derived human liver stem/progenitor cells infused 3 days postsurgery improve liver regeneration in a mouse model of extended hepatectomy. Cell transplantation 26(2): 351-364.

9. Kordes C, Sawitza I, Götze S, Häussinger D (2015) Beyond fibrosis: stellate cells as liver stem cells. Z Gastroenterol 53(12): 1425-1431.

10. Lotfy, Ahmed, Mohamed Salama, Faten Zahran, Elena Jones, et al. (2014) Characterization of mesenchymal stem cells derived from rat bone marrow and adipose tissue: a comparative study. Int J stem cells $7(2): 135-142$.

11. Dominici M, Le Blanc K, Mueller I (2006) Minimal criteria for defining multipotent mesenchymal stromal cells. The International Society for Cellular Therapy position statement. Cytotherapy 8(4) 315-317.
12. Barry EP, Boynton RE, Haynesworth S, Murphy JM, Zaia J (1999) The monoclonal antibody $\mathrm{SH}-2$, raised against human mesenchymal stem cells, recognizes an epitope on endoglin (CD105). Biochem Biophys Res Commun 265(1): 134-139.

13. Ode A, Kopf J, Kurtz A (2011) CD73 and CD29 concurrently mediate the mechanically induced decrease of migratory capacity of mesenchymal stromal cells. European Cells and Materials 22: 26-42.

14. Eckle T, Fullbier L, Wehrmann M (2007) Identification of ectonucleotidases CD39 and CD73 in innate protection during acute lung injury. J Immunol 178(12): 8127-8137.

15. Haeryfar SMM, Hoskin DW (2004) Thy-1: more than a mouse pan T cell marker. J Immunol 173(6): 3581-3588.

16. Williams AF, Gagnon J (1982) Neuronal cell Thy-1 glycoprotein: homology with immunoglobulin. Science 216(4547): 696-703.

17. Najimi M, Khuu DN, Lysy PA, Jazouli N, Abarca J, et al. (2007) Adult-derived human liver mesenchymal-like cells as a potential progenitor reservoir of hepatocytes?. Cell transplantation 16(7): 717-728.

18. Rozemuller H, Prins HJ, Naaijkens B, Staal J, Buhring HJ, et al. (2010) Prospective isolation of mesenchymal stem cells from multiple mammalian species using cross-reacting anti-human monoclonal antibodies. Stem Cells and Development 19(12): 1911-1921.

19. Delorme B, Ringe J, Gallay N (2008) Specific plasma membrane protein phenotype of culture-amplified and native human bone marrow mesenchymal stem cells. Blood 111(5): 2631-2635.

20. Noort WA, Oerlemans MI, Rozemuller H (2012) Human versus porcine mesenchymal stromal cells: phenotype, differentiation potential, immunomodulation and cardiac improvement after transplantation. J Cell Mol Med 16(8): 1827-1839.

21. Kumar A, Dudhal S, Sunkara M, Usman H, Varshney A, et al. (2016) Dopaminergic-primed fetal liver mesenchymal stromal-like cells can reverse parkinsonian symptoms in 6-hydroxydopamine-lesioned mice. Cytotherapy 18(3): 307-319.

22. Pelekanos RA, Li J, Gongora M, (2012) Comprehensive transcriptome and immunophenotype analysis of renal and cardiac MSC-like populations supports strong congruence with bone marrow MSC despite maintenance of distinct identities. Stem Cell Research 8(1): 58-73.

23. Payushina OV, Butorina NN, Sheveleva ON, Kozhevnikova MN, Starostin (2013) Cell Composition of the Primary Culture of Fetal Liver. Bull Exp Biol Med 154(4): 566-573.

24. Jones EA, Crawford A, English A (2008) Synovial fluid mesenchymal stem cells in health and early osteoarthritis: detection and functional evaluation at the single-cell level. Arthritis Rheum 58(6): 1731-1740.

25. Taofeng Lu, Pengfei Hu, Xiaohua Su, Changli Li, Yuehui Ma, et al. (2014) Isolation and characterization of mesenchymal stem cells derived from fetal bovine liver. Cell Tissue Bank 15(3): 439-450.

26. Mu R, Bian YC, Pu YB, Li XC, Wang FL, et al. (2013) Isolation and biological characterization of mesenchymal stem cells from Beijing fatty chicken fetal liver. Hereditas35(3): 365-372.

27. Luo X, Gupta K, Ananthanarayanan A, Wang Z, Xia L, et al. (2018) Directed Differentiation of Adult Liver Derived Mesenchymal Like Stem Cells into Functional Hepatocytes. Scientific reports 8(1): 2818.

28. Herrera Sanchez MB, Maria Beatriz Herrera, (2014) Human liver stem cells and derived extracellular vesicles improve recovery in a murine model of acute kidney injury. Stem cell research \& therapy 5(6): 124"?

29. Fonsato V, Collino F, Herrera MB, Cavallari C, Deregibus MC, et al. (2012) Human liver stem cell-derived microvesicles inhibit hepatoma growth in SCID mice by delivering antitumor microRNAs. Stem cells 30(9): 1985-1998. 
30. Herrera MB, Bruno S, Buttiglieri S, Tetta C, Gatti S, et al. (2006) Isolation and characterization of a stem cell population from adult human liver. Stem cells 24(12): 2840-2850.

31. Wang Y, Yu X, Chen E, Li L (2016) Liver-derived human mesenchymal stem cells: a novel therapeutic source for liver diseases. Stem cell res ther 7(1): 71 .

32. Lee JH, Park HJ, Kim YA, Lee DH, Noh JK, (2012) The phenotypic characteristic of liver-derived stem cells from adult human deceased donor liver. Transplantation proceedings 44(4): 1110-1112.

33. Yang HM, Cho MR, Sung JH, Yang SJ, Nam MH (2011) The Effect of Human Fetal Liver-Derived Mesenchymal Stem Cells on CD34+ Hematopoietic Stem Cell Repopulation in NOD/Shi-scid/IL-2Rãnull Mice. Transplant proc 43(5).

34. Kholodenko IV, Kholodenko RV, Manukyan GV, Burunova VV, Yarygin KN (2016) Mesenchymal-epithelial transition in culture of stromal progenitor cells isolated from the liver of a patient with alcoholic cirrhosis. Bulletin of experimental biology and medicine 162(1): 115119.

35. Giuliani M, Fleury M, Vernochet A, Ketroussi F, Clay D (2011) Long-lasting inhibitory effects of fetal liver mesenchymal stem cells on T-lymphocyte proliferation. PLoS One 6(5): e19988.

36. Raicevic G, Najar M, Najimi M, El Taghdouini A, Van Grunsven LA, et al. (2015) Influence of inflammation on the immunological profile of adult-derived human liver mesenchymal stromal cells and stellate cells. Cytotherapy 17(2): 174-85.
37. Götherström C, Ringden O, Westgren M, Tammik C, Le Blanc K (2003) Immunomodulatory effects of human foetal liver-derived mesenchymal stem cells. Bone marrow transplant 32(3): 265-272.

38. Liu D, Yovchev MI, Zhang J, Alfieri AA, Tchaikovskaya T, et al. (2015) Identification and characterization of mesenchymal-epithelial progenitor-like cells in normal and injured rat liver. Am J Pathol 185(1): $75110-75128$

39. Tanaka M, Itoh T, Tanimizu N, Miyajima A (2011) Liver stem/progenitor cells: their characteristics and regulatory mechanisms. The Journal of Biochemistry 149(3): 231-239.

40. Conigliaro A, Colletti M, Cicchini C, Guerra MT, Manfredini R, et al. (2008) Isolation and characterization of a murine resident liver stem cell. Cell death and differentiation 15(1): 123-133.

41. Pan Q, Fouraschen SM, Kaya FS, Verstegen MM, Pescatori M (2011) Mobilization of hepatic mesenchymal stem cells from human liver grafts. Liver Transpl 17(5): 596-609.

42. Rafael Moreno, Itziar Martínez González, Marta Rosal, Abduljalil Farwati, Eduard Gratacós et al. (2010) Characterization of Mesenchymal Stem Cells Isolated from the Rabbit Fetal Liver. Stem Cell Dev 19(10): 1579-1588.

43. Weiss MC, Strick Marchand H (2003) Isolation and characterization of mouse hepatic stem cells in vitro. In Seminars in liver disease 23(4): 313-324.

\section{Your next submission with Juniper Publishers will reach you the below assets}

- Quality Editorial service

- Swift Peer Review

- Reprints availability

- E-prints Service

- Manuscript Podcast for convenient understanding

- Global attainment for your research

- Manuscript accessibility in different formats

( Pdf, E-pub, Full Text, Audio)

- Unceasing customer service

Track the below URL for one-step submission https://juniperpublishers.com/online-submission.php 